\title{
Características sociodemográficas y su influencia en el uso de Tecnologías de Información en Chile ${ }^{1}$
}

\author{
Enrique Canessa \\ Universidad Adolfo Ibáñez, Viña del Mar, Chile. \\ Email: ecanessa@uai.cl \\ José Maldifassi \\ Universidad Adolfo Ibáñez, Viña del Mar, Chile. \\ Email: jose.maldifassi@uai.cl \\ Ariel Quezada \\ Universidad Adolfo Ibáñez, Viña del Mar, Chile. \\ Email: ariel.quezada@uai.cl
}

\begin{abstract}
Resumen: Este estudio examina la relación entre características sociodemográficas de las personas y su uso de Tecnologías de Información (TI) en Chile, empleando una versión abreviada del Modelo de Aceptación de Tecnología, TAM (Technology Acceptance Model, por su sigla en inglés). Los datos recolectados apoyan al TAM, mostrando que tanto la facilidad de uso percibida (FUP) como la utilidad percibida (UP) impactan positivamente en el uso de computadores (U). A su vez, la facilidad de uso favorece la utilidad percibida. Dentro de las variables sociodemográficas, el ingreso familiar (IF) y nivel educacional (NE) influyen favorablemente en FUP, UP y el uso (U) de computadores, al contario de la edad (E), la cual impacta negativamente en dichas variables. Asimismo, se advirtió que existe una correlación positiva entre ingreso familiar y nivel educacional, y una correlación negativa entre edad y NE. A diferencia de otros estudios, esta investigación recolectó datos de una muestra heterogénea con sujetos de diferentes ingresos familiares, edades y niveles educacionales, aproximándose a la población urbana chilena. Por ello, este estudio presenta una panorámica del uso de TI, de utilidad para guiar políticas gubernamentales enfocadas en la promoción del uso colectivo de TI en un país en desarrollo.
\end{abstract}

Palabras clave: TAM, características sociodemográficas, uso de computadores, país en desarrollo

\section{Socio-demographic characteristics and their influence on the use of Information Technologies in Chile}

\footnotetext{
Abstract; This paper examines the relationship between people's sociodemographic characteristics and their use of Information Technology (IT) in Chile, employing an abridged version of the Technology Acceptance Model (TAM). The data collected supports the TAM, showing that the perceived easiness of use $(\mathrm{PEOU})$ and perceived usefulness (PU) positively impact the use of computers
} 
(U). In turn, perceived ease of use favors perceived usefulness. Regarding the socio-demographic variables, family income (FI) and educational level (EL) positively influence PEOU, PU and U, contrary to age (A), which negatively impacts those variables. Likewise, the data showed a positive correlation between FI and EL, and a negative correlation between A and EL. Unlike previous studies, this research gathered data using a heterogeneous sample, which included subjects who exhibited different income, age, and educational levels, approximating the urban Chilean population. Thus, this paper presents a picture of the use of IT, which makes it useful for guiding government policy making aimed at promoting the collective use of IT in a developing country.

Key words: TAM, socio-demographic characteristics, computer use, developing country

\section{Características sociodemográficas e sua influência sobre o uso da TI no Chile}

Resumo: Este estudo examina a relação entre as características sociodemográficas dos indivíduos eo uso de Tecnologia da Informação (TI) no Chile, usando uma versão abreviada do Modelo de Aceitação de Tecnologia, TAM. Os dados coletados apoiam a TAM, mostrando que tanto a facilidade de uso percebida (FUP) e utilidade percebida (PU) têm um impacto positivo sobre o uso de computadores (U). Por sua vez, facilidade de uso promove a utilidade percebida. Entre as variáveis _ sócio-demográficas, a renda familiar (IF) e nível de escolaridade (NE) influenciam favoravelmente FUP, UP e use (U) de computadores, para dizer a idade (E), que tem um impacto negativo sobre essas variáveis. Também foi observado que há uma correlação positiva entre renda familiar e nível educacional e uma correlação negativa entre idade e NE. Diferentemente de outros estudos, esta pesquisa colheu dados de uma amostra heterogênea com sujeitos de renda familiar diferente, idade e níveis de ensino, aproximando-se da população chilena urbana. Portanto, este estudo apresenta uma visão geral do uso de TI, útil para orientar as políticas governamentais voltadas para a promoção do uso coletivo de TI em um país em desenvolvimento.

Palavras-chave: TAM, características sociodemográficas, uso de computadores, países em desenvolvimento

$* * *$

\section{Introducción}

Actualmente, la adopción y uso eficiente de las Tecnologías de Información (TI), tanto a nivel personal como organizacional, son aspectos claves para mantener el crecimiento económico y una buena calidad de vida en un país (Jeyaraj, Rottman y Lacity, 2006; Lu, 2001; WEF, 2009). En particular, en las naciones en vías de desarrollo, las TI son un factor importante para alcanzar los aspectos mencionados (Brown y Licker, 2003; Tallon y Kraemer, 1999). Desafortunadamente el uso de las TI en dichas naciones no está ampliamente difundido, lo que ha creado una marcada diferencia en el empleo de las TI entre países desarrollados y en vías de desarrollo. A este fenómeno se le ha denominado "brecha digital” (Lu, 2001), la cual los países en desarrollo han tratado de disminuir mediante el incremento del uso de las TI. 
Siendo Chile un país en vías de desarrollo, la mayoría de sus ciudadanos aún enfrentan múltiples barreras económicas, personales, educacionales, familiares, culturales y organizacionales para poder adoptar y usar TI (PNUD, 2006). Es por esto, que el presente estudio emplea el Modelo de Aceptación de Tecnología (TAM, Technology Acceptance Model, por su sigla en inglés) (Davis, 1989), para analizar algunas variables sociodemográficas que pueden explicar las barreras que las personas enfrentan para poder hacer uso de las TI. Se ha seleccionado el TAM como base para este análisis, ya que ha recibido un amplio y fuerte respaldo empírico por muchos estudios previos (McCoy, Galletta y King, 2007; Yousafzai, Foxall y Pallister, 2007a). Aunque el TAM ha sido empleado en investigaciones efectuadas en países en vías de desarrollo (Abdul-Gader, 1992; Dias, 1998; McCoy, Galletta y King, 2007; Mostafa, 2006; Yousafzai et al., 2007a), este se ha aplicado principalmente en naciones de habla inglesa (Al-Gahtani, 2001; Segars y Grover, 1993; Yousafzai et al., 2007a). Experiencias en idioma castellano se encuentran en López y López (2006) evaluando a una muestra de universitarios españoles. Por su parte, en Iberoamérica el TAM fue empleado en un estudio del uso de TI por gerentes en Brasil (Dias, 1998), pero con una reducida cantidad de sujetos (70 gerentes) y enfocado en instituciones financieras. Posteriormente, en una investigación de la aplicabilidad del TAM en varios países, se evaluó a una muestra reducida incluyendo entre 30 y 9 estudiantes universitarios de México, Argentina y Brasil (McCoy et al., 2007). Las conclusiones de dichos estudios son que el TAM es generalmente aplicable. No obstante, la reducida cantidad de sujetos participantes, los que provenían de organizaciones y contextos específicos (universidades e instituciones financieras), amenazan la generalización de sus hallazgos. Por el contrario, dado que la presente investigación intenta entregar una visión amplia, incluyendo personas de muy diferentes edades, niveles económicos y educacionales, se emplea una muestra heterogénea y relativamente grande. Adicionalmente este estudio permite analizar la aplicabilidad del TAM en un país en desarrollo de habla hispana, particularmente Chile.

\section{Modelo de Aceptación de Tecnología y formulación de las hipótesis}

El TAM (Davis, 1989) en su versión abreviada postula que un usuario de un sistema o equipo computacional (TI) lo empleará más frecuentemente en la medida que lo perciba como fácil de usar y le resulte útil. A su vez, la facilidad de uso influirá positivamente en la percepción de utilidad. En una versión modificada del TAM (Davis, Bagozzi y Warshaw, 1989) se observa que algunas variables externas pueden influir en dichas percepciones (ver Figura 1). Como el presente estudio integra en el análisis algunas variables externas, se ocupará dicho modelo, el cual ha sido usado y respaldado por varios estudios anteriores (Adams, Nelson y Todd, 1992; Igbaria, Zinatelli, Cragg y Cavaye, 1997; Straub, Limayen y Karahanna-Evaristo, 1995; Szajna, 1996). 


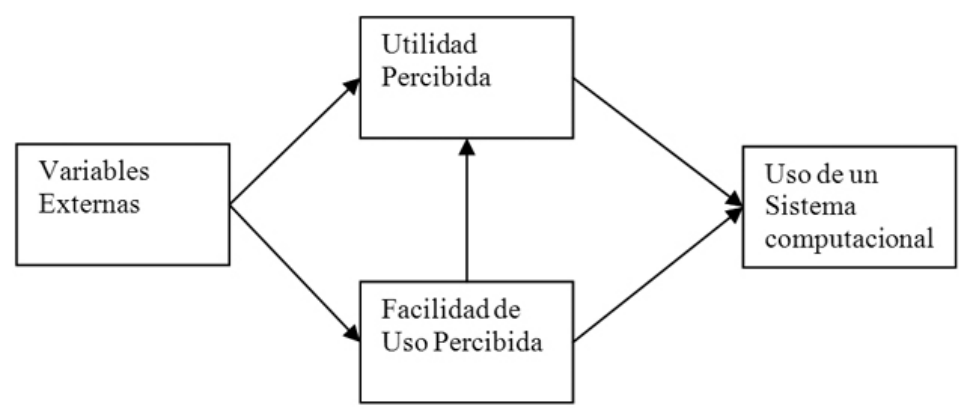

Figura 1. Versión abreviada del Modelo de Aceptación de Tecnología (TAM).

Davis (1989) entiende como utilidad percibida de un sistema computacional a la probabilidad subjetiva que tiene una persona en cuanto a que el uso de dicho sistema incrementará su rendimiento laboral. Por facilidad de uso percibida se alude al grado en que una persona cree que usar un sistema particular exigirá un bajo esfuerzo. En el caso del presente estudio, las variables externas (sociodemográficas) corresponden a nivel educacional, edad y nivel de ingreso familiar de un individuo. A continuación, se establecerá las relaciones entre esas variables externas y aquellos factores del TAM mostrados en la Figura 1, las que originan las hipótesis correspondientes.

En cuanto a las variables externas (sociodemográficas) que inciden en el uso individual de Internet, Bimber (2001) estableció que la educación y el ingreso económico de cada sujeto explicaban el uso de dicha tecnología. Asimismo, Agarwal y Prasad (1999) y el meta-análisis de Yousafzai et al. $(2007 a, b)$ hallaron que el nivel educacional de un sujeto impactaba su percepción de utilidad de las TI y su uso. A medida que el nivel educacional de un individuo aumenta, éste probablemente efectuará tareas más elaboradas, lo cual podría impulsarlo a hacer un mayor uso de las TI y demostrarle su utilidad en su quehacer. Por otra parte, una persona con mejor educación, poseerá habilidades y conocimientos, tales como habilidades lectoras, de escritura y conocimientos matemáticos, que le facilitarán el uso del equipamiento computacional. Estos argumentos permiten establecer el siguiente grupo de hipótesis:

H1a: A mayor nivel educacional (NE) de una persona, mayor será su facilidad de uso percibida (FUP) de computadores.

H1b: A mayor nivel educacional (NE) de una persona, mayor será su utilidad percibida (UP) de computadores.

H1c: A mayor nivel educacional (NE) de una persona, mayor será su uso (U) de computadores.

Otra variable sociodemográfica relacionada es la edad de una persona. Con su acelerada tasa de innovación, las TI demandan de sus usua- 
rios un significativo grado de alfabetismo computacional (computer literacy) para poder ocuparlas beneficiosamente (PNUD, 2006). Adicionalmente, estudios muestran que las personas más jóvenes tienden a percibir las TI como más útiles y fáciles de usar que individuos de mayor edad (Maldifassi y Canessa, 2009; Morris y Venkatesh, 2000). A su vez, McCloskey (2006) encontró que a mayor edad de una persona menor es su percepción de facilidad de uso de sistemas de ventas por Internet y su frecuencia de uso. Al igual que en otros países en vías de desarrollo, en Chile la introducción de TI a un nivel general ha ocurrido recientemente (PNUD, 2006), pudiendo existir una cantidad considerable de adultos mayores que siguen efectuando sus labores habituales sin mayor uso de TI y que tienen un alfabetismo computacional bastante bajo. Por ello, podemos formular las siguientes hipótesis:

H2a: A mayor edad de una persona (E), menor será su facilidad de uso percibida (FUP) de computadores.

H2b: A mayor edad de una persona (E), menor será su utilidad percibida (UP) de computadores.

H2c: A mayor edad de una persona (E), menor será su uso (U) de computadores.

Por otra parte, en cuanto a la variable sociodemográfica ingreso económico familiar (IF), los individuos con mayores ingresos tienen un mayor acceso a todo tipo de bienes, incluyendo computadores. Aún más, una persona de un país en vías de desarrollo, en comparación con otra de un país desarrollado, requiere un porcentaje mayor de sus ingresos para adquirir equipamiento computacional (WEF, 2009). Por lo tanto, se puede afirmar que las personas con mayores ingresos tendrán más acceso a equipamiento computacional y, consecuentemente, tenderán a usar las TI con mayor frecuencia. Por otro lado, en Latinoamérica la fuerte segmentación socioeconómica ha generado importantes diferencias en la calidad educacional y de infraestructura entre estratos altos y bajos (CEPAL, 2008). En particular, quienes acceden a una educación de alta calidad están más preparados para usar efectiva y productivamente las TI, percibiéndolas como útiles y fáciles de usar. Asimismo, esta mejor educación probablemente les permitirá acceder a mejores trabajos, más tecnológicos y/o más demandantes intelectualmente, en los que requerirá ocupar una importante cantidad y variedad de herramientas TI, reforzando la percepción de utilidad de las TI. Esto se refleja en las siguientes hipótesis:

H3a: A mayor ingreso familiar (IF) de una persona, mayor será su facilidad de uso percibida (FUP) de computadores.

H3b: A mayor ingreso familiar (IF) de una persona, mayor será su utilidad percibida (UP) de computadores.

H3c: A mayor ingreso familiar (IF) de una persona, mayor será su uso (U) de computadores.

Como ya se ha mencionado, muchos estudios previos han corroborado las relaciones entre uso, utilidad y facilidad de uso percibidas postuladas por el TAM (Yousafzai et al., 2007a,b). Por eso, basados en el TAM 
podemos formular las siguientes hipótesis:

H4: A mayor la facilidad de uso percibida (FUP) de computadores por una persona, mayor será su utilidad percibida (UP) de los mismos.

H5: A mayor la facilidad de uso percibida (FUP) de computadores por una persona, mayor será su uso (U) de los mismos.

H6: A mayor la utilidad percibida (UP) de computadores por una persona, mayor será su uso (U) de los mismos.

Finalmente y no siendo el foco de este estudio, también se tuvo la oportunidad de indagar otras relaciones adicionales en cuanto a nivel educacional y edad. Se ha descrito que en países en vías de desarrollo los adultos mayores tienden a tener una menor escolaridad, dado que sólo recientemente se ha ido elevando la cobertura de la enseñanza pública en dichas naciones (CEPAL, 2008). A su vez, del mismo estudio se puede inferir que en estratos socioeconómicos altos se lograrán mayores niveles de escolaridad. Por lo tanto, se sugieren los siguientes enunciados:

H7: A mayor edad de un individuo (E), menor será su nivel educacional (NE).

H8: A mayor ingreso familiar (IF) de un individuo, mayor será su nivel educacional (NE).

La Figura 2 muestra esquemáticamente las relaciones entre variables articuladas en las hipótesis planteadas. Cabe destacar que para este estudio el uso de un sistema computacional se refiere a su uso a nivel personal, variable análoga a la utilizada en otros estudios y nominada como "computación personal” (personal computing) (Bagozzi, Davis y Warshaw, 1992; Delone y Mclean, 1992; Igbaria et al., 1997; Raymond, 1985).

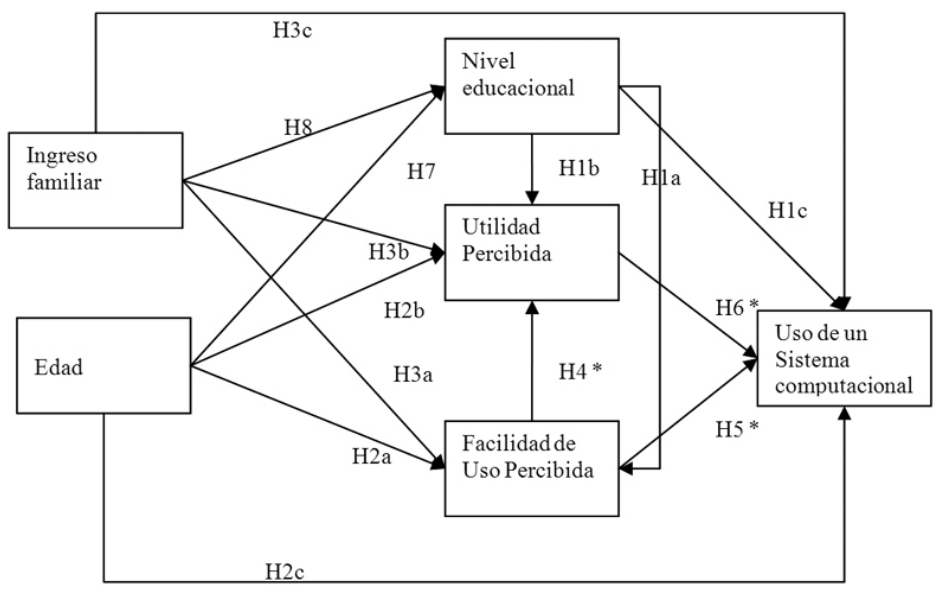

Nota: Las hipótesis marcadas con un asterisco corresponden al TAM abreviado

Figura 2. Modelo de Aceptación de Tecnología (TAM) abreviado con las variables externas consideradas en este estudio. 


\section{Metodología e instrumento de medición}

Para recolectar los datos del estudio se realizó una encuesta que mide las variables presentadas en la Figura 2. En el caso de facilidad de uso y utilidad percibida, se usaron los ítemes desarrollados por Davis (1989), traduciéndolos al español (ver Apéndice A). Por esto, FUP se midió usando 4 ítemes y UP con 5 ítemes, ambos empleando una escala Likert de 5 puntos. Estos instrumentos también han sido traducidos a otros lenguajes manteniendo buenas propiedades evaluativas (Abdul-Gader, 1992; Kamel y Hussein, 2002; López y López, 2006; Mostafa, 2006; Tsai, Lin y Tsai, 2001). Anticipando que parte de los sujetos a encuestar tendrían un bajo nivel educacional, se agregó algunas clarificaciones a ciertos ítemes que potencialmente presentarían problemas de entendimiento. Dichos ítemes están relacionados con conceptos de eficiencia, eficacia y productividad, acercando los ítemes originales a un lenguaje más accesible para el común de las personas.

Tomando en cuenta el tamaño muestral relativamente grande y la heterogeneidad de los encuestados, fue necesario medir la frecuencia de uso de computadores preguntándola en una escala Likert desde 1 (nunca) a 5 (siempre) (ver detalle en Apéndice A). Los adjetivos usados en dicha escala son una traducción al español de los recomendados por Bass, Cascio y O’Connor (1974). Debemos hacer notar que el autoreporte del uso de un sistema podría no reflejar fielmente el uso real, pero estudios previos sugieren que es una medida de uso relativo apropiada (Bagozzi et al., 1992; Blair y Burton, 1987; Davis et al., 1989). Además, como este estudio mide muchas variables independientes usando autoreporte de valores percibidos en vez de reales, el medir la variable dependiente de la misma forma puede ayudar a disminuir los errores asociados al método empleado (Harrison, McLaughlin y Coatler, 1996).

Respecto a la medición de ingreso familiar, se preguntó a cada encuestado por su ingreso familiar mensual total en ocho rangos (ver Apéndice A), los cuales representan adecuadamente a los estratos socio-económicos chilenos usados en estudios de censo y mercadeo, según lo practica INE (2003) y Collect (2007). Estas mismas fuentes sugieren el uso de rangos, en lugar de preguntar directamente por el monto del ingreso, ya que las personas suelen sentirse poco confortables al revelar sus ingresos económicos.

El nivel educacional de cada encuestado se midió solicitando que cada persona reportara la cantidad de años aprobados en educación básica, media, técnica y universitaria, clasificación utilizada por el Ministerio de Educación de Chile (MINEDUC, 2009). Para transformar esos datos a nivel educacional, se elaboró una escala de conversión de intervalos aproximadamente iguales, la que se muestra en la Tabla 1. 
Tabla 1: Tabla de conversión de años de enseñanza aprobados a nivel educacional

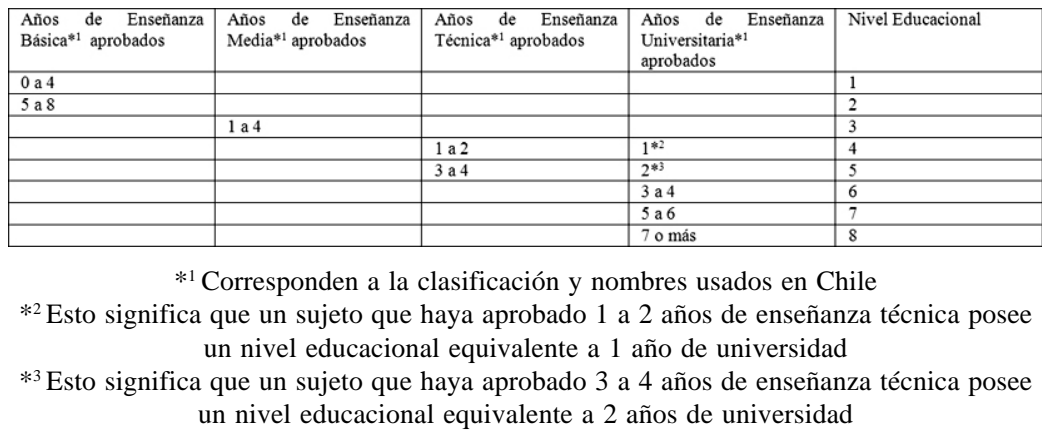

La tabla de conversión fue elaborada dado que no existe una medida de transformación de años de escolaridad a nivel educacional, según el Ministerio de Educación de Chile (MINEDUC). Para su elaboración se contó con la participación de dos expertos en educación y fue visada por una representante del MINEDUC para efectos de este estudio.

En cuanto al autoreporte de la edad del encuestado, para evitar la deseabilidad social, se preguntó por la fecha de nacimiento de éste en vez de su edad.

Al contar con una primera versión del cuestionario, se recurrió a dos expertos con alto nivel de inglés y a un angloparlante para verificar la calidad de su traducción. Con esta versión se efectuó una evaluación piloto con 15 personas de niveles educacionales medios a bajos para asegurar su comprensión en el segmento más crítico de la muestra. El resultado de lo anterior motivó cambios estilísticos menores.

La versión definitiva del cuestionario fue administrada por encuestadores quienes contactaban a los participantes para que individual y voluntariamente lo completaran. Se aplicó en una muestra por conveniencia en lugares de amplia concurrencia pública en las ciudades más pobladas de Chile: Santiago, Valparaíso, Viña del Mar, Concepción y Antofagasta. Estas ciudades concentran casi el 70\% de la población chilena (INE, 2003), por lo que la muestra corresponde principalmente a población urbana. La recolección de datos tomó aproximadamente dos semanas y se obtuvo 570 encuestas.

\section{Resultados y prueba de las hipótesis}

Los datos obtenidos con los 570 cuestionarios fueron sometidos a un análisis estadístico descriptivo que evidenció una distribución aproximadamente normal de éstos, sin sesgos hacia los extremos de las escalas 
utilizadas. La Tabla 2 muestra los estadísticos correspondientes y la matriz de correlación de los datos. Para facilitar la lectura de los resultados, en el Apéndice B se entrega una breve descripción conceptual de las pruebas estadísticas empleadas.

Tabla 2: Matriz de correlaciones y estadísticos descriptivos de los datos recolectados

\begin{tabular}{|l|l|l|l|l|l|l|l|l|l|l|l|l|l|}
\hline & NE & UP1 & UP2 & UP3 & UP4 & UP5 & FUP1 & FUP2 & FUP3 & FUP4 & USO & IF & EDAD \\
\hline NE & 1.000 & & & & & & & & & & & & \\
\hline UP1 & 0.468 & 1.000 & & & & & & & & & & & \\
\hline UP2 & 0.496 & 0.930 & 1.000 & & & & & & & & & & \\
\hline UP3 & 0.497 & 0.917 & 0.916 & 1.000 & & & & & & & & & \\
\hline UP4 & 0.482 & 0.890 & 0.895 & 0.909 & 1.000 & & & & & & & & \\
\hline UP5 & 0.431 & 0.800 & 0.818 & 0.830 & 0.840 & 1.000 & & & & & & & \\
\hline FUP1 & 0.410 & 0.717 & 0.719 & 0.727 & 0.701 & 0.689 & 1.000 & & & & & & \\
\hline FUP2 & 0.427 & 0.723 & 0.737 & 0.740 & 0.726 & 0.706 & 0.913 & 1.000 & & & & & \\
\hline FUP3 & 0.416 & 0.652 & 0.678 & 0.671 & 0.655 & 0.645 & 0.844 & 0.845 & 1.000 & & & & \\
\hline FUP4 & 0.400 & 0.699 & 0.729 & 0.721 & 0.708 & 0.683 & 0.894 & 0.903 & 0.864 & 1.000 & & & \\
\hline USO & 0.562 & 0.717 & 0.730 & 0.739 & 0.739 & 0.653 & 0.711 & 0.709 & 0.691 & 0.703 & 1.000 & & \\
\hline IF & 0.584 & 0.412 & 0.440 & 0.441 & 0.425 & 0.378 & 0.354 & 0.375 & 0.344 & 0.366 & 0.500 & 1.000 & \\
\hline EDAD & -0.195 & -0.456 & -0.473 & -0.457 & -0.471 & -0.401 & -0.495 & -0.504 & -0.455 & -0.485 & -0.495 & -0.123 & 1.000 \\
\hline PROM. & 4.744 & 3.481 & 3.475 & 3.504 & 3.432 & 3.424 & 3.363 & 3.300 & 2.933 & 3.261 & 2.905 & 3.937 & 42.415 \\
\hline D.EST. & 1.908 & 1.370 & 1.393 & 1.387 & 1.394 & 1.351 & 1.362 & 1.344 & 1.375 & 1.344 & 1.486 & 2.456 & 17.742 \\
\hline MIN & 1 & 1 & 1 & 1 & 1 & 1 & 1 & 1 & 1 & 1 & 1 & 1 & 16 \\
\hline MAX & 8 & 5 & 5 & 5 & 5 & 5 & 5 & 5 & 5 & 5 & 5 & 8 & 91 \\
\hline
\end{tabular}

$$
\mathrm{N}=570
$$

A continuación, se realizó un análisis factorial exploratorio para los datos correspondientes a FUP y UP, usando el método de extracción de factores por máxima verosimilitud y con rotación varimax (Pedhazur y Schmelkin, 1991). Se extrajeron dos factores, los que dan cuenta del $90.5 \%$ de la varianza de los datos. La rotación varimax mostró una buena separación de los ítemes constitutivos de cada uno de los dos factores (ver Tabla 3).

Tabla 3: Análisis factorial exploratorio de los ítemes correspondientes a los factores FUP y UP, extracción máxima verosimilitud, rotación varimax

\begin{tabular}{|lll|}
\hline \multicolumn{3}{|l|}{ Factor } \\
\hline & $\mathbf{1}$ & $\mathbf{2}$ \\
\hline UP1 & $\mathbf{0 . 8 7 0}$ & 0.398 \\
UP2 & $\mathbf{0 . 8 6 2}$ & 0.422 \\
UP3 & $\mathbf{0 . 8 6 2}$ & 0.423 \\
UP4 & $\mathbf{0 . 8 4 4}$ & 0.412 \\
UP5 & $\mathbf{0 . 7 4 2}$ & 0.445 \\
FUP1 & 0.425 & $\mathbf{0 . 8 4 8}$ \\
FUP2 & 0.442 & $\mathbf{0 . 8 4 7}$ \\
FUP3 & 0.383 & $\mathbf{0 . 8 1 0}$ \\
FUP4 & 0.421 & $\mathbf{0 . 8 5 0}$ \\
\hline
\end{tabular}


Como muestra la Tabla 3, solamente el ítem UP5 muestra una menor separación comparado con el resto de los ítems. Sin embargo los coeficientes alfa de Cronbach calculados para cada factor mostraron una apropiada confiabilidad interna. Para la escala de FUP el coeficiente es 0.97 y para UP es 0.96, ambos muy por encima del valor mínimo sugerido de 0.70 (Nunally, 1978).

Posteriormente, se usó modelamiento de ecuaciones estructurales (SEM, Structural Equation Modeling, por su sigla en inglés) (Bagozzi, 1994; Bollen, 1989) para verificar el modelo y las hipótesis. Empleando el paquete estadístico Lisrel (Joresborg y Sorborn, 1996), se construyó un modelo inicial (M1), usando todos los indicadores para IF, Edad, NE, FUP, UP y Uso. El ajuste del modelo con los datos fue inadecuado, ya que el estadístico $\mathrm{c}^{2}=97.14(\mathrm{gl}=52)$ y su valor observado de significancia = 0.00015 así lo sugieren (Hu y Bentler, 1999; Joresborg y Sorborn, 1996; Pedhazur y Schmelkin, 1991). Indagando en las probables causas de esto, se advirtió un alto índice de modificación (13.6) correspondiente a la correlación entre los errores (residuos) de los indicadores UP5 y FUP1, violando la suposición que tales errores deben ser independientes (Pedhazur y Schmelkin, 1991) y, en consecuencia, sugiriendo que uno de esos dos indicadores podría presentar algún problema.

En efecto, el ítem UP5 exhibe una mala separación de factores, por lo que es plausible que éste sea la causa de la alta correlación entre residuos. Por otro lado, ya en la evaluación piloto de la encuesta se había anticipado que dicho ítem pudiera representar una dificultad para que las personas lo entendieran. Es factible que para personas con un bajo nivel educacional les sea bastante difícil interpretar correctamente expresiones del tipo: "Usar los computadores me provee de información que podría permitirme tomar mejores decisiones”. Una correcta evaluación de expresiones similares implica un nivel de abstracción relativamente elaborado. Por ello, se removió el ítem UP5 del modelo y se calculó un nuevo modelo (M2). Este modelo obtuvo un estadístico $\mathrm{c}^{2}=73.4(\mathrm{gl}=42)$ con un valor observado de significancia igual a 0.0019. La diferencia entre estadísticos $c^{2}$ entre ambos modelos mostró que hubo un mejor calce de M2 con los datos respecto a M1 (M1: $c^{2}=97.14 ; g l=52$ y M2: $\left.c^{2}=73.4 ; g l=42\right)$, sugiriendo que dicho cambio fue acertado.

Los resultados de la aplicación de la encuesta piloto también sugirieron posibles problemas en el indicador FUP3 ("Es/sería fácil para mi convertirme en un usuario avanzado de los computadores”). Para algunas personas podría ser complejo reconocer qué es un usuario avanzado y qué tan difícil sería convertirse en uno de ellos. Por eso, se removió el ítem FUP3 y se calculó un nuevo modelo (M3), cuyo estadístico c ${ }^{2}=66.5$ ( $\mathrm{gl}=$ 33) y valor observado de significancia $=0.00048$, sugirió un mal calce. Finalmente, un índice de modificación igual a 26.7 para la correlación entre los residuos de los ítemes UP2 y UP3 mostraba que todavía se podía perfeccionar el modelo. Analizando dichos ítemes, se aprecia que en UP2 ("Usar los computadores aumenta mi productividad en mis actividades dia- 
rias (puedo hacer más rápido las cosas)”), el concepto de productividad es abstracto y podría causar problemas de entendimiento. Pese a que esto se había detectado y se agregó una explicación entre paréntesis, dicho ítem probó ser difícil de entender por lo que se decidió removerlo y, en consecuencia, calcular un nuevo modelo (M4).

El nuevo modelo (M4) dio un estadístico $c^{2}=25.0(g l=24)$ con un valor observado de significancia $=0.404$. Otros índices de ajuste también mostraron un buen calce entre el modelo y los datos. El error cuadrático medio de aproximación (RMSEA, Root Mean Square Error of Approximation por su sigla en inglés) fue igual a 0.009, muy por debajo del valor recomendado de 0.06, el GFI $=0.99$, AGFI $=0.98$, NFI y NNFI $=$ 1.00, todos ellos sobre el valor sugerido de 0.95 (Hu y Bentler, 1999; Joresborg y Sorborn, 1996; Pedhazur y Schmelkin, 1991). Por ello, se concluyó que el modelo M4 exhibía un buen calce con los datos, el cual se aprecia en la Figura 3.

Además de los índices anteriores, el gráfico Cuartil-Cuartil (Q-Q) de los residuos -no mostrado- revela que estos se distribuyen normalmente, permitiendo la evaluación de significación estadística de los coeficientes del modelo. También se calculó el coeficiente alfa de Cronbach para los ítemes que miden FUP (ítemes FUP1, FUP2 y FUP4) y UP (ítemes UP1, UP3 y UP4), dando 0.97 en ambos casos, sobre el valor 0.70 recomendado por Nunally (1978). Lo anterior es consecuente con coeficientes entre los indicadores y sus respectivos factores cercanos a 1.0 y estadísticamente significativos (ver ls en Figura 3).

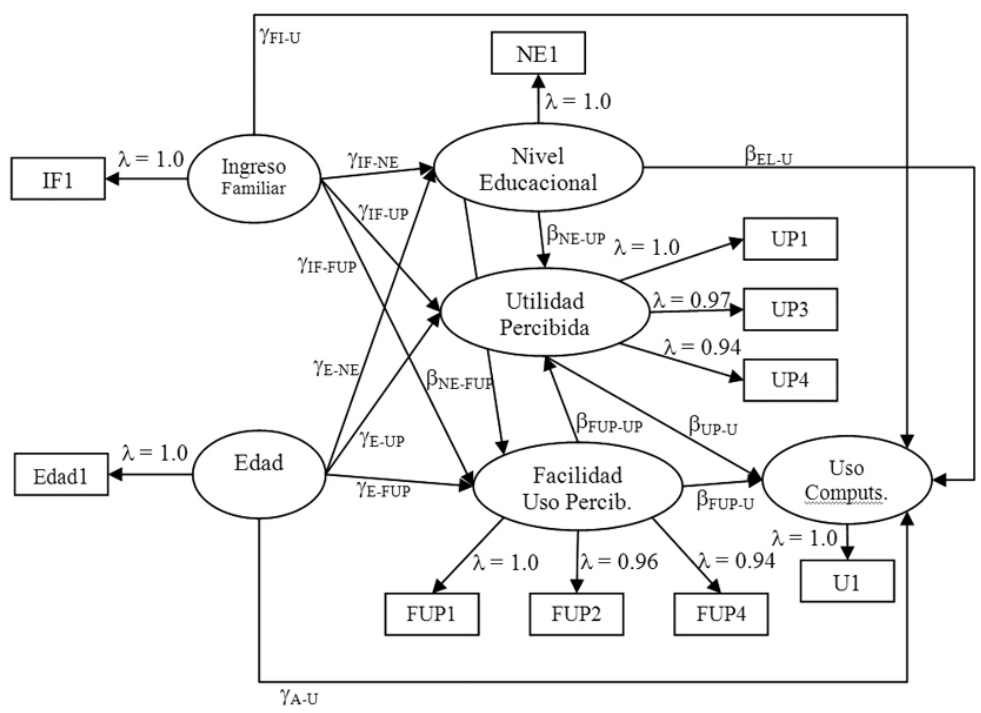

Figura 3. Modelo estructural final para uso de computadores (M4). 
Obtenido un modelo adecuado, se procedió a usar sus resultados para verificar las hipótesis. La Tabla 4 presenta los principales resultados del modelo, donde los coeficientes gama y beta corresponden a los mostrados en la Figura 3.

\section{Tabla 4: Síntesis de resultados del modelo estructural para la verificación de las hipótesis}

\begin{tabular}{|c|c|c|c|c|c|}
\hline & \multicolumn{2}{|l|}{ Coeficientes Gama } & \multicolumn{3}{|l|}{ Coeficientes Beta } \\
\hline & $\begin{array}{l}\text { Ingreso Familiar } \\
\text { (IF) }\end{array}$ & Edad (E) & $\begin{array}{ll}\begin{array}{l}\text { Nivel } \\
(\mathrm{NE})\end{array} & \text { Educacional } \\
\end{array}$ & \begin{tabular}{|l|} 
Facilidad Uso \\
Percibida (FUP)
\end{tabular} & $\begin{array}{l}\text { Utilidad Percibida } \\
\text { (UP) }\end{array}$ \\
\hline Nivel Educacional (NE) & $0.44\left(\gamma_{\mathrm{IFNNE}}\right)$ & $-0.01\left(\gamma_{\mathrm{ENE}}\right)$ & & & \\
\hline Facilidad Uso Perc. (FUP) & $0.10\left(\gamma_{\text {IFFFUP }}\right)$ & $-0.03\left(\gamma_{\text {E.FUP }}\right)$ & $0.16\left(\beta_{\text {NE.FUP }}\right)$ & & \\
\hline Utilidad Percibida (UP) & $0.06\left(\gamma_{\text {IF }-U P}\right)$ & $-0.01\left(\gamma_{\mathrm{E}-\mathrm{UP}}\right)$ & $0.10\left(\beta_{\mathrm{NE} \text {.UP }}\right)$ & $0.63\left(\beta_{\text {EUP.UP }}\right)$ & \\
\hline Uso $(U)$ & $0.08\left(\gamma_{\mathrm{IIF}-\mathrm{U}}\right)$ & $-0.01\left(\gamma_{\mathrm{E}-\mathrm{U}}\right)$ & $0.13\left(\beta_{\mathrm{NE} \cdot \mathrm{U}}\right)$ & $0.33\left(\beta_{\text {FUP.U. }}\right)$ & $0.38\left(\beta_{\text {UP.U.U }}\right)$ \\
\hline
\end{tabular}

Todos los valores son estadísticamente significativos al menos a 0.01

Empleando los resultados de la Tabla 4, se verificó si los datos respaldan a cada hipótesis formulada. La Tabla 5 presenta dicho análisis.

\section{Tabla 5: Resumen de la verificación de las hipótesis}

\begin{tabular}{|c|c|c|}
\hline Hipótesis & Resultados (ver Tabla 4) & Observaciones \\
\hline H1a: A mayor NE, mayor FUP & $\begin{array}{l}\text { Aceptada: coeficiente beta NE - FUP } \\
\text { positivo y e.s. }\end{array}$ & $\begin{array}{l}\text { Resultado coincidente con Pijpers, } \\
\text { Bemelmans, Heemstra y van Montfort } \\
(2001)\end{array}$ \\
\hline H1b: A mayor NE, mayor UP & $\begin{array}{l}\text { Aceptada: coeficiente beta NE - UP } \\
\text { positivo y e.s. }\end{array}$ & $\begin{array}{l}\text { Resultado coincidente con Pijpers et al. } \\
(2001) \\
\text { Impacto } \mathrm{NE} \rightarrow \mathrm{FUP}>\mathrm{NE} \rightarrow \mathrm{UP}\left(\gamma_{\mathrm{NE}-\mathrm{FUP}}\right. \\
\left.=0.16>\gamma_{\mathrm{NE} \cdot \mathrm{UP}}=0.10\right) \text {. }\end{array}$ \\
\hline H1c: A mayor NE, mayor U & $\begin{array}{l}\text { Aceptada: coeficiente beta NE-U positivo } \\
\text { y e.s. }\end{array}$ & $\begin{array}{l}\text { Resultado coincidente con Pijpers et al. } \\
(2001)\end{array}$ \\
\hline H2a: A mayor E, menor FUP & $\begin{array}{l}\text { Aceptada: coeficiente gama E - FUP } \\
\text { negativo y e.s. }\end{array}$ & $\begin{array}{l}\text { Resultado coincidente con Pijpers et al. } \\
(2001) \\
\text { Impacto } \mathrm{E} \rightarrow \mathrm{FUP}>\mathrm{E} \rightarrow \mathrm{NE} \\
\left(\left|\gamma_{\mathrm{E}-\mathrm{FUP}}\right|=0.03>\left|\gamma_{\mathrm{E}-\mathrm{NE}}\right|=0.01\right)\end{array}$ \\
\hline H2b: A mayor E, menor UP & $\begin{array}{l}\text { Aceptada: coeficiente gama E - UP } \\
\text { negativo y e.s. }\end{array}$ & $\begin{array}{l}\text { Resultado coincidente con Pijpers et al. } \\
(2001) \\
\text { Impacto } \mathrm{E} \rightarrow \mathrm{FUP}>\mathrm{E} \rightarrow \mathrm{UP} \\
\left(\left|\gamma_{\mathrm{E}-\mathrm{FUP}}\right|=0.03>\left|\gamma_{\mathrm{E}-\mathrm{UP}}\right|=0.01\right)\end{array}$ \\
\hline $\mathrm{H} 2 \mathrm{c}$ : A mayor E, menor U & $\begin{array}{l}\text { Aceptada: coeficiente gama E-U negativo } \\
\text { y e.s. }\end{array}$ & $\begin{array}{l}\text { Resultado contradice a Pijpers et al. } \\
\text { (2001). Se puede deber a que ese estudio } \\
\text { se realizó en un pais desarrollado con } \\
\text { ejecutivos seniors y gran experiencia en } \\
\text { el uso de TI, mientras en este estudio la } \\
\text { muestra es más heterogénea. }\end{array}$ \\
\hline H3a: A mayor IF, mayor FUP & $\begin{array}{l}\text { Aceptada: coeficiente gama IF - FUP } \\
\text { positivo y e.s. }\end{array}$ & $\begin{array}{l}\text { Impacto IF } \rightarrow \text { NE }>\text { IF } \rightarrow \text { FUP } \\
\left(\gamma_{\text {IF }, N E}=0.44>\gamma_{\text {IF }, \text {, UP }}=0.10\right)\end{array}$ \\
\hline H3b: A mayor IF, mayor UP & $\begin{array}{l}\text { Aceptada: coeficiente gama IF - UP } \\
\text { positivo y e.s. }\end{array}$ & $\begin{array}{l}\text { Impacto IF } \rightarrow \text { FUP }>\text { IF } \rightarrow \text { UP } \\
\left(\gamma_{\text {F-FUP }}=0.10>\gamma_{\text {F-UP }}=0.06\right)\end{array}$ \\
\hline H3c: A mayor IF, mayor U & $\begin{array}{l}\text { Aceptada: coeficiente gama IF - U positivo } \\
\text { y e.s. }\end{array}$ & \\
\hline H4: A mayor FUP, mayor UP & $\begin{array}{l}\text { Aceptada: coeficiente beta FUP - UP } \\
\text { positivo y e.s. }\end{array}$ & $\begin{array}{l}\text { Este coeficiente es el más grande del } \\
\text { modelo, confirmando el meta-análisis del } \\
\text { TAM de Yousafzai et al. ( } 2007 \mathrm{~b})\end{array}$ \\
\hline H5: A mayor FUP, mayor U & $\begin{array}{l}\text { Aceptada: coeficiente beta FUP }-\mathrm{U} \\
\text { positivo y e.s. }\end{array}$ & $\begin{array}{l}\text { Resultado coincidente con Pijpers et al. } \\
(2001)\end{array}$ \\
\hline H6: A mayor UP, mayor U & $\begin{array}{l}\text { Aceptada: coeficiente beta UP-U positivo } \\
\text { y e.s. }\end{array}$ & $\begin{array}{l}\text { Impacto UP } \rightarrow \mathrm{U}>\text { FUP } \rightarrow \mathrm{U} \\
\left(\beta \text { up.U }=0.38>\beta_{\text {Fup.U }}=0.33\right) \text {. Ambos } \\
\text { resultados coinciden con el meta-análisis } \\
\text { del TAM de Yousafzai et al. }(2007 \mathrm{~b})\end{array}$ \\
\hline H7: A mayor E, menor NE & $\begin{array}{l}\text { Aceptada: coeficiente gama } E-N E \\
\text { positivo y e.s. }\end{array}$ & \\
\hline H8: A mayor IF, mayor NE & $\begin{array}{l}\text { Aceptada: coeficiente gama IF }-\mathrm{NE} \\
\text { positivo y e.s. }\end{array}$ & \\
\hline
\end{tabular}

Nota: e.s. $=$ Estadísticamente Significativo al menos al nivel 0.01 
En la Tabla 5 se puede apreciar que los datos respaldan a las hipótesis. Asimismo, el porcentaje de varianza explicada por las ecuaciones estructurales fue $\mathrm{R}^{2}=0.36$ para NE, $\mathrm{R}^{2}=0.41$ para FUP, $\mathrm{R}^{2}=0.68$ para UP y $\mathrm{R}^{2}=0.69$ para U. Así, se puede afirmar que el modelo ayuda a explicar un adecuado porcentaje de la varianza de las variables dependientes.

\section{Discusión, limitaciones e implicancias prácticas de los resultados}

Como muestra la verificación de las hipótesis, el presente estudio respalda al TAM abreviado, lo que muestra que dicho modelo se puede emplear adecuadamente en el contexto latinoamericano y en países en desarrollo. Específicamente se comprobó que la facilidad de uso percibida impacta positivamente en la percepción de utilidad y en el uso de computadores. Al mismo tiempo, se observó que la utilidad percibida incentiva el uso de esta tecnología. Por otra parte, las variables sociodemográficas ingreso familiar y nivel educacional -externas al TAM e incorporadas en este estudio- favorecen la facilidad de uso y la utilidad percibida, lo que consecuentemente incrementa el uso de computadores. En el caso de la edad, las personas mayores tienden a percibir a los computadores como difíciles de usar y menos útiles en sus actividades cotidianas. Esto último podría explicarse ya que los datos indican que las personas de edades más avanzadas se asocian con aquellas de menores niveles educacionales. A su vez, el estudio evidenció que el nivel educacional se correlaciona positivamente con el ingreso familiar de una persona.

Si bien es cierto que las relaciones entre las variables del TAM ya han sido ampliamente respaldadas por numerosos estudios previos, esta investigación difiere en tres aspectos importantes.

Primero, el presente trabajo es uno de los primeros que adapta, desarrolla y verifica un instrumento en español para medir la percepción de facilidad de uso y utilidad de TI adaptado para personas con distintas características sociodemográficas. Segundo, la muestra de este estudio incluyó sujetos de diferentes niveles educacionales, ingresos económicos y edades, a diferencia de la mayoría de los estudios del TAM en los cuales participaron principalmente personas con formación profesional y pertenecientes a empresas o universidades (López y López, 2006; Yousafzai et al., 2007a, b). Dado que en esta investigación la recolección de datos fue realizada en las principales ciudades de Chile, sus resultados son preferentemente aplicables al uso de TI en población urbana chilena y no se limitan a una organización en particular. Tercero, este estudio es uno de los primeros que analiza el uso de TI en un país latinoamericano en desarrollo usando el TAM (Yousafzai et al., 2007a, b), a excepción del análisis de Dias (1998) en Brasil, pero limitado a una pequeña cantidad de gerentes de instituciones financieras, y una exploración de la aplicabilidad del TAM en varios países, incluyendo 
entre 9 y 30 estudiantes universitarios de México, Argentina y Brasil (McCoy et al., 2007).

Como en toda investigación, este estudio también presenta limitaciones. Se experimentaron algunos problemas con la interpretación de algunos ítemes del instrumento para medir facilidad de uso y utilidad percibida. Pese a que dichos ítemes fueron mejorados aún son perfectibles. En cuanto a la medición de uso de computadores, el dato registrado fue el autoreporte que las personas tienen sobre su empleo en lugar de una medición del uso real. Sin embargo, estudios previos han demostrado que el uso de esta variable sustituta es adecuado para los fines de esta investigación (Bagozzi et al., 1992; Blair y Burton, 1987; Davis et al., 1989). Finalmente, dadas algunas limitaciones operativas del equipo de investigación, el presente estudio empleó una muestra de conveniencia, en lugar de haber usado otro procedimiento más formal. Pese a ello, el tamaño muestral fue apropiado (570 personas).

A partir de este estudio se pueden inferir algunas implicancias prácticas. En esta investigación se aplicó el cuestionario a una muestra en las ciudades que concentran el $70 \%$ de la población chilena (INE, 2003). Dicha muestra la integraron personas de diversas edades, niveles de ingreso económico y niveles educacionales. Así, se pueden sugerir algunas consideraciones para mejorar políticas públicas tendientes a incrementar el uso productivo de las TI e Internet en contextos urbanos, lo que coincide con uno de los objetivos de los últimos gobiernos chilenos (PNUD, 2006). En este sentido, este estudio muestra que personas con mayores ingresos económicos tienden a hacer un mayor uso de computadores, dado que para éstas la adquisición de dichos bienes involucra una proporción menor de sus recursos comparado con las personas de menores ingresos. Por esto, medidas gubernamentales como la entrega de computadores portátiles a estudiantes talentosos de escasos recursos y dotar a las escuelas públicas de computadores conectados a Internet, están bien enfocadas a nivelar el acceso a las TI. Aunque estas medidas acercan dichas tecnologías a quienes cuentan con menores ingresos, aún muchas personas de bajos recursos carecen de las habilidades para aplicar productivamente las TI a sus actividades y trabajo (Maldifassi y Canessa, 2009; WEF, 2009). Sumado a lo anterior, este estudio evidencia que el nivel educacional influye positivamente sobre la utilidad con que se percibe a las TI y sobre su facilidad para usarlas. Por lo tanto, los esfuerzos gubernamentales para la mejora progresiva de la cobertura y calidad de la enseñanza pública deberán tener un impacto positivo sobre la utilidad y facilidad en el uso de las TI y, en consecuencia, repercutirá positivamente en la adopción y uso de dichas tecnologías, tal como lo demuestra la presente investigación y el TAM.

Reforzando las acciones anteriores, el gobierno podría llevar a cabo iniciativas complementarias que ayuden a incrementar la utilidad y facilidad de uso de las TI. En Chile, una acción podría consistir en que las personas interactuaran con las agencias de desarrollo social a través de Internet, especialmente aquellas de menores recursos y nivel educacional, ya que 
éstas son quienes hacen uso intensivo de los servicios brindados por dichas agencias. Por ello, permitir y fomentar su interacción a través de Internet podría ahorrarles recursos (costo de transporte, tiempo de espera para ser atendidas, etc.) (Strelitz y Lister, 2008), lo que aumentaría la percepción de utilidad de las TI y, a futuro, esto podría inducir su aplicación a actividades más elaboradas y posiblemente para usos aún más productivos. Aunque no todos los servicios pueden ser implementados a través de Internet, algunas tareas básicas, como el ingreso de datos personales y postulaciones a beneficios sociales podrían ser realizados por los usuarios vía Internet. La implementación de centros de Internet con un grupo de funcionarios que asistan a los usuarios les ayudaría a familiarizarse con dicha interacción. En el desarrollo de dichos centros, debería diseñarse cuidadosamente los sistemas y sus interfaces para que sean fáciles de usar por gente mayor y de escasa educación, aumentando su percepción de facilidad de uso, contribuyendo a una mejor adopción de la tecnología. Estos centros tendrían el beneficio adicional de permitir incluir a personas que ya no están dentro del sistema educacional.

Por otra parte, este estudio muestra que las personas de edad avanzada tienden a percibir a las TI como difíciles de usar y con utilidad limitada para ellos, lo que redunda en su poco empleo. Dado que el segmento de chilenos sobre 60 años en la actualidad corresponde a un 15\% de la población, el que a mediados del siglo XXI aumentará a un 30\% (INE, 2003), sería conveniente implementar acciones para ayudar a que dichas personas incrementen su uso de las TI. Al igual que en el caso de personas de menores ingresos económicos, el uso más intensivo de TI permitirá a los adultos mayores economizar recursos en sus trámites u otras actividades cotidianas. Sería apropiado, entonces, que los adultos mayores se mantengan vigentes y actualizados en el manejo de las TI por dos situaciones: a) progresivo incremento de actividades y servicios que operan por Internet y, b) el rápido desarrollo que experimentan las TI. Luego, una persona que hoy realiza una cierta cantidad de operaciones vía Internet, en un futuro podría desperdiciar el potencial de esta TI si solamente se mantiene haciendo dichas operaciones, desaprovechando la vasta oferta de aplicaciones que podrían llegar a existir. Asimismo, alguien que en la actualidad hace un buen uso de las TI, si no mantiene al día sus conocimientos informáticos, podría quedar desactualizado en el futuro. Por lo tanto, para prevenir que los adultos mayores queden rezagados de los avances en TI, sería provechoso promover una actualización permanente. Para ello podrían usarse los mismos centros de Internet ya descritos, asegurándose que provean capacidades acordes con las necesidades particulares de los adultos mayores.

Revisadas las implicancias prácticas derivadas del análisis de las variables del TAM, este estudio también sugiere que el modelo -como un todo- puede ser empleado adecuadamente en países en vías de desarrollo e hispano parlantes. Aún más, podría generarse nuevas investigaciones tendientes a refinar el uso del TAM, indagando con mayor profundidad cómo las variables sociodemográficas afectan a los constructos del TAM (Bagozzi, 2007; Straub y Burton-Jones, 2007; Venkatesh, Davis y Morris, 2007) y/o 
integrando otras variables ya usadas en estudios previos (Venkatesh, Morris, Davis y Davis, 2003; Venkatesh y Davis, 2000; Yi, Wu y Tung, 2006). Sin embargo, el enfoque anterior tiene sus límites, puesto que tiende a obtener miradas parceladas de un fenómeno social. Por ello, una alternativa complementaria sugeriría un enfoque más global e interpretivista al estudio de la adopción y uso de TI (Lamb y Kling, 2003; Silva, 2007).

Finalmente, el desarrollo de esta investigación ha permitido dar luces sobre cómo el uso y adopción de TI se manifiesta en un contexto urbano en Latinoamérica y en relación a variables sociodemográficas. La utilidad que reviste este trabajo reside en las posibilidades de profundización del análisis, dado que la indagación de otras variables sobre el uso de TI se ve favorecida por la aplicabilidad del TAM en dicho contexto. A su vez, este conocimiento permitirá mejorar las políticas públicas que ayuden a países con características similares a reducir la brecha digital, uno de los aspectos claves para alcanzar el desarrollo de las naciones.

\section{Agradecimientos}

Agradecemos la cooperación de Filadelfo de Mateo y Héctor Allende, de la Universidad Adolfo Ibáñez, en el desarrollo del instrumento de medición y en el diseño del procedimiento de conversión de años de escolaridad a nivel educacional. También agradecemos a María Elena Villalobos del Ministerio de Educación, Chile, por el tiempo que invirtió en verificar dicho procedimiento. 


\section{Apéndice A}

\section{Instrumento de medición}

Para medir Utilidad Percibida (UP) y Facilidad de Uso Percibida (FUP), se usó el instrumento original desarrollado por Davis (1989), el que fue traducido al español. Davis (1989) define UP como "the prospective user's subjective probability that using a specific application system will increase his or her job performance within an organizational context" y FUP como "refers to the degree to which a person believes that using a particular system would be free of effort”. Se agregaron algunas explicaciones/clarificaciones para hacer el instrumento más entendible por personas de bajo nivel educacional. Los ítemes son los siguientes:

UP1: Usar los computadores mejora el rendimiento en mis actividades diarias (puedo hacer más cosas)

UP2: Usar los computadores aumenta mi productividad en mis actividades diarias (puedo hacer más rápido las cosas)

UP3: Encuentro que los computadores son útiles en mis actividades diarias (me ayuda a hacer las cosas)

UP4:Usar los computadores mejora mi efectividad en mis actividades diarias (puedo hacer mejor las cosas)

UP5:Usar los computadores me provee de información que podría permitirme tomar mejores decisiones

FUP1: Aprender a usar los computadores es/sería fácil para mi

FUP2: Encuentro/encontraría fácil usar los computadores para hacer lo que yo desee

FUP3: Es/sería fácil para mi convertirme en un usuario avanzado de los computadores

FUP4: Yo encuentro/encontraría los computadores fáciles de usar

Todos los ítemes se contestan en una escala Likert de 5 puntos, donde 1 = totalmente en desacuerdo, $2=$ en desacuerdo, $3=$ ni de acuerdo ni en desacuerdo, 4 = de acuerdo, 5 = totalmente de acuerdo

Para medir uso, se empleó la siguiente pregunta:

Indique que tan frecuentemente Usted hace uso de los computadores en sus actividades diarias. Este ítem se contesta usando una escala Likert de 5 puntos, donde $1=$ nunca, 2 = ocasionalmente, 3 = bastantes veces, 4 $=$ muy seguido, 5 = siempre

Para medir los años de educación/escolaridad aprobados se usó la siguiente pregunta:

Por favor indique lo siguiente: años):

Años totales de enseñanza básica y media aprobados (máximo 12

Años totales de enseñanza técnica aprobados (máximo 4 años):

Años totales de enseñanza universitaria aprobados (máximo 12 años): 
Para medir ingreso familiar mensual total, se usó la siguiente escala:

$$
\begin{aligned}
& 1=\text { menos de } \$ 200.000 \text { mensuales } \\
& 2=\text { entre } \$ 200.000 \text { y } \$ 500.000 \text { mensuales } \\
& 3=\text { entre } \$ 501.000 \text { y } \$ 800.000 \text { mensuales } \\
& 4=\text { entre } \$ 801.000 \text { y } \$ 1.100 .000 \text { mensuales } \\
& 5=\text { entre } \$ 1.101 .000 \text { y } \$ 1.400 .000 \text { mensuales } \\
& 6=\text { entre } \$ 1.401 .000 \text { y } \$ 1.700 .000 \text { mensuales } \\
& 7=\text { entre } \$ 1.701 .000 \text { y } \$ 2.000 .000 \text { mensuales } \\
& 8=\text { más de } \$ 2 \text { millones mensuales }
\end{aligned}
$$

Nota: \$ corresponde a Pesos Chilenos, tasa de cambio app. 1 USD $=\$ 530$ (Junio, 2009) 


\section{Apéndice B}

\section{Herramientas Estadísticas Empleadas en el Análisis de Datos}

A continuación, se entrega una breve descripción conceptual de las herramientas estadísticas utilizadas en el artículo, ordenadas según su aparición en el texto principal. Las descripciones entregadas no son matemáticamente rigurosas sino que apuntan a una comprensión didáctica de los conceptos.

a) Análisis factorial exploratorio: herramienta estadística que separa un conjunto de ítemes que miden diversos factores. Estos ítemes conforman grupos que miden un solo factor al mismo tiempo. Para cada ítem el análisis calcula un coeficiente que indica qué tan bien mide el ítem a cada factor, con un valor máximo 1.0 (el factor es exactamente medido por el ítem) a un mínimo 0.0 (el factor no es medido por el ítem). Existen diferentes formas para calcular cada factor y sus ítemes asociados. La más usada es emplear el Método de Extracción de factores por máxima verosimilitud y con rotación varimax. Consiste en identificar una cantidad de factores tal que maximice la probabilidad de que dichos factores existan en el fenómeno estudiado. Luego, se calcularán los coeficientes tratando de que sean lo más altos posibles para los ítemes que miden un cierto factor y lo más bajos posibles para el resto. Esto hará que exista una mínima cantidad de factores y con los ítemes que los miden lo más separados posible.

b) Coeficientes Alfa de Cronbach: es una medida que indica la consistencia interna de un conjunto de ítemes que miden un factor. Su rango va de 1.0, lo que indica que el conjunto de ítemes mide al factor que representan con máxima consistencia; a 0.0, indicando que el conjunto de ítemes no mide en absoluto al factor que representan.

c) Modelamiento de Ecuaciones Estructurales (SEM, Structural Equation Modeling, por su sigla en inglés): es una herramienta que calcula la asociación (regresión) de variables independientes sobre variables dependientes. Por ejemplo, se podría modelar la influencia de coeficiente intelectual (CI) sobre rendimiento académico (RA). Dado que el CI de una persona no puede ser medido directamente, se denomina variable latente, la que se medirá indirectamente a través de variables indicadoras, las que sí pueden ser medidas directamente. Los coeficientes 1 indican qué tan bien cada variable indicadora mide a la variable latente respectiva. A su vez, los coeficientes g describen la relación o influencia entre variables latentes independientes. Finalmente, los coeficientes b reflejan la relación o influencia entre una variable latente dependiente con una independiente. Estos tres coeficientes miden la fuerza de la relación entre variables, teniendo valores que van de 1.0 (relación perfecta 
entre variables) a 0.0 (no existe relación alguna entre variables).

d) Estadístico $\mathrm{c}^{2}$ : es una medida que evalúa la existencia de una diferencia apreciable entre los valores de dos variables o, por el contrario, si dicha diferencia es solamente fruto del azar. En general, mientras más grande el valor de $c^{2}$, existe mayor evidencia que de dicha diferencia realmente existe. Lo anterior se ve reflejado en el valor observado de significancia, que es la probabilidad de que una diferencia entre el valor de dos variables ocurra sólo por azar. Luego, a menor valor observado de significancia para la diferencia, más evidencia existe de que dicha diferencia sea real. En el caso del SEM, el estadístico $c^{2}$, mide la diferencia que existe entre los datos medidos y el modelo planteado (hipotético). Dado que se busca que el modelo hipotético refleje lo más fielmente posible a los datos, se pretende obtener un modelo con el valor más bajo posible de $\mathrm{c}^{2}$, y por ende, con el mayor valor posible de valor observado de significancia.

e) Índice de modificación: es el valor en que el estadístico $c^{2}$ disminuiría si las relaciones entre variables del modelo se modificaran, incluyéndolas o excluyéndolas del modelo. El SEM entrega dichos índices, los que se pueden emplear para mejorar el calce de un modelo. Para ello se debe cambiar la relación entre variables del modelo hipotético según el valor de dichos índices.

f) GFI, AGFI, NFI y NNFI y Error cuadrático medio de aproximación (RMSEA, Root Mean Square Error of Approximation por su sigla en inglés): en general corresponden a índices de bondad de ajuste de un modelo SEM a los datos recolectados. Reflejan qué tan bien el modelo hipotético se ajusta a dichos datos. Para no extender demasiado este apéndice evitaremos describir cada índice pero, en general, GFI, AGFI, NFI y NNFI están basados en el valor de $c^{2}$. Mientras más pequeños sean sus valores, mejor será el ajuste del modelo a los datos. Por su parte el RMSEA es el promedio de los cuadrados de las diferencias residuales entre los datos recolectados y el modelo hipotético. En consecuencia, mientras más pequeño sea el valor de RMSEA, mejor será el calce entre datos y modelo.

g) Gráfico Cuartil-Cuartil (Q-Q) de los residuos: es una gráfica que muestra los residuos que existen entre los datos y el modelo, reflejando qué tan bien se distribuyen estos en relación a una distribución normal de probabilidades.

h) $\mathbf{R}^{\mathbf{2}}$ (coeficiente de determinación): indica la proporción de la variabilidad observada en los datos recolectados que es explicada por el conjunto de variables independientes incluidas en el modelo. Un $\mathrm{R}^{2}$ igual a 1.0, significa que toda la variabilidad observada en los datos es explicada por dichas variables, mientras que un valor igual a 0.0 , indica que nada de la variabilidad es explicada por las variables independientes, con lo cual el modelo no es útil. 


\section{Nota}

${ }^{1}$ JEL Classification

O100 - Economic Development: General

O140 - Industrialization; Manufacturing and Service Industries; Choice of Technology 


\section{Bibliografía}

Abdul-Gader, A.H. (1992), End-User Computing Success Factors: Further Evidence from a Developing Nation. Journal of End User Computing, 4(3), 4-13.

Adams, D.A., Nelson, R.R. y Todd, P.A. (1992), Perceived Usefulness, Ease of Use, and Usage of Information Technology: A Replication. MIS Quarterly, 16(2), 227-247.

Al-Gahtani, S. (2001), The applicability of TAM outside North America: An empirical test in the United Kingdom. Information Resources Management Journal, 14(3), 37-46.

Agarwal, R. y Prasad, J. (1999), Are individual differences germane to the acceptance of new information technologies? Decision Sciences, 30(2), 361-391.

Bagozzi, R., Davis, F. y Warshaw, P. (1992), Development and Test of a Theory of Technological Learning and Usage. Human Relations, 45(7), 659-685.

Bagozzi, R. (1994), Structural Equation Models in Marketing Research: Basic Principles. En R.P. Bagozzi (ed.). Principles of Marketing Research (pp. 317-385), Oxford: Blackwell.

Bagozzi, R. (2007), The Legacy of the Technology Acceptance Model and a Proposal for a Paradigm Shift. Journal of the Association for Information Systems, 8(4), 244-254.

Bass, B., Cascio, W. y O’Connor, E. (1974), Magnitude estimations of expressions of frequency and amount, Journal of Applied Psychology, 59(3), 313-320.

Bimber, B. (2001), Information and Political Engagement in America: the Search for Effects of Information Technology at the Individual Level. Political Research Quarterly, 54(1), 53-67.

Blair, E. y Burton, S. (1987), Cognitive Process Used by Survey Respondents to Answer Behavioral Frequency Questions. Journal of Consumer Research, 14, 280-288.

Bollen, K. A. (1989), Structural Equations with Latent Variables. New York: John Wiley \& Sons Inc.

Brown, I. y Licker, P. (2003), Exploring Differences in Internet Adoption and Usage between Historically Advantaged and Disadvantaged Groups in South Africa. Journal of Global Information Technology Management, 6(3), 6-26. 
Collect Investigaciones de Mercado S.A. (2007, 16 de marzo), Disponible en: http://www.collect.cl/admnoticias/admin/estudios_disponibles/ GSE\%20Base\%20Censo\%202002.pdf

Comisión Económica para América Latina y el Caribe (CEPAL) (2008), Calidad de la educación: las desigualdades más allá del acceso y la progresión educativa. Santiago de Chile: Naciones Unidas

Davis, F.D. (1989), Perceived usefulness, perceived ease of use, and user acceptance of technology. MIS Quarterly 13(3), 319-340.

Davis, F.D., Bagozzi, R.P. y Warshaw, P.R. (1989), User Acceptance of Computer Technology: a Comparison of Two Theoretical Models. Management Science, 35(8), 982-1003.

Delone, W. y McLean, E. (1992), Information Systems Success: The Quest for the Dependent Variable. Information Systems Research, 31, 60-95.

Dias, D. D. S. (1998), Managers' motivation for using information technology. Industrial Management \& Data Systems, 98(7), 338-42.

Harrison, S., McLaughlin, M.E. y Coalter, T.M. (1996). Context, cognition, and common method variance: Psychometric and verbal evidence. Organizational Behavior and Human Decision Processes, 68, 246-261.

Hu, L. y Bentler, P.M. (1999), Cutoff Criteria for Fit Indices in Covariance Structure Analysis: Conventional Criteria versus New Alternatives. Structural Equation Modeling, 6, 1-55.

Igbaria, M., Zinatelli, N., Cragg, P. y Cavaye, A.L.M. (1997), Personal Computing Acceptance Factors in Small Firms: A Structural Equation Model. MIS Quarterly, 21(3), 279-302.

Instituto Nacional de Estadísticas de Chile (INE) (2003), Censo 2002. Síntesis de Resultados. Santiago de Chile: Instituto Nacional de Estadísticas.

Jeyaraj, A., Rottman, J. W. y Lacity, M. C. (2006), A review of the predictors, linkages, and biases in IT innovation adoption research. Journal of Information Technology, 21: 1-23.

Joresborg, K. y Sorborn, D. (1996), Lisrel 8: User's reference guide. Chicago: Scientific Software International.

Kamel, S. y Hussein, M. (2002), The emergence of e-commerce in a developing nation: Case of Egypt. Benchmarking: An International Journal, 9, 146-153.

Lamb, R. y Kling, R. (2003), Reconceptualizing users as social actors in information systems research. MIS Quarterly 27(2), 197-235. 
López, L. M. y López, J. M. (2006), Estudio comparado de las estimaciones de dos versiones del modelo de aceptación de la tecnología (TAM) mediante los programas AMOS y PLS. Investigaciones Europeas de Dirección y Economía de la Empresa, 12 (3), pp. 95-110.

Lu, M. (2001), Digital divide in developing countries. Journal of Global Information Technology Management, 4(3), 1.

Maldifassi, J. y Canessa, E. (2009), Information technology in Chile: How perceptions and use are related to age, gender, and social class. Technology in Society, 31, 273-286

McCloskey, D. W. (2006), The Importance of Ease of Use, Usefulness, and Trust to Online Consumers: An Examination of the Technology Acceptance Model with Older Consumers. Journal of Organizational and End User Computing, 18(3), 47-65.

McCoy, S., Galletta, D. F. y King, W. R. (2007), Applying TAM across cultures: the need for caution. European Journal of Information Systems, 16, 81-90.

Ministerio de Educación de Chile (MINEDUC) (2009, 6 de abril), Niveles Educacionales. Disponible en: http://600.mineduc.cl/informacion/ info_nive/index.php

Morris, M. G. y Venkatesh, V. (2000), Age Differences in Technology Adoption Decisions: Implications for a Changing Workforce. Personnel Psychology, 53, 375-403.

Mostafa, M. M. (2006), An Empirical Investigation of Egyptian Consumers Usage Patterns and Perceptions of the Internet. International Journal of Management, 23(2), 243-260.

Nunally, J. (1978), Psychometric Theory. New York: McGraw Hill.

Pedhazur, E. y Schmelkin, L. (1991), Measurement, design and analysis: an integrated approach. New Jersey: Lawrence Erlbaum Associates.

Pijpers, G.M., Bemelmans, T.M.A., Heemstra, F. J. y van Montfort, K. A.G.M. (2001), Senior executives' use of information technology. Information and Software Technology, 43, 959-971.

Programa de las Naciones Unidas para el Desarrollo (PNUD) (2006), Desarrollo Humano en Chile: las nuevas tecnologías ¿un salto al futuro?, Santiago de Chile: Programa de las Naciones Unidas para el Desarrollo (PNUD).

Raymond, L. (1985), Organizational Characteristics and MIS Success in the Context of Small Business, MIS Quarterly, 9(1): 37-52. 
Segars, A. H. y Grover, V. (1993), Re-examining perceived ease of use and usefulness: A confirmatory factor analysis. MIS Quarterly, 17, 517-525.

Silva, L. (2007), Post-positivist Review of Technology Acceptance Model. Journal of the Association for Information Systems, 8(4), 255-266.

Straub, D. W. y Burton-Jones, A. (2007), Veni, Vidi, Vici: Breaking the TAM Logjam. Journal of the Association for Information Systems, 8(4), 223-229.

Straub, D., Limayen, M. y Karahanna-Evaristo, E. (1995), Measuring System Usage: Implications for IS Theory Testing. Management Science, 41(8), 1328-1342.

Szajna, B. (1996), Empirical Evaluation of the Revised Technology Acceptance Model. Management Science, 42(1), 85-92.

Tallon, P. P. y Kraemer, K. L. (1999), Information Technology and Economic Development: Ireland's Coming of Age with Lessons for Developing Countries. University of California, Center for Research on Information Technology and Organizations, Paper 136, eScholarship repository.

Tsai, C., Lin, S. y Tsai, M. (2001), Developing an Internet attitude scale for high school students. Computers and Education, 37, 41-51.

Strelitz, J. y Lister, R. (2008). Why Money Matters. Family income, poverty and children's lives. London: Save the Children.

Venkatesh, V. y Davis, F. D. (2000), A Theoretical Extension of the Technology Acceptance Model: Four Longitudinal Field Studies. Management Science, 46(2), 186-204.

Venkatesh, V., Morris, M.G., Davis, G. B. y Davis, F. D. (2003), User acceptance of information technology: Toward a unified view. MIS Quarterly, 27(3), 425-478.

Venkatesh, V., Davis, F. y Morris, M. G. (2007), Dead Or Alive? The Development, Trajectory and Future Of Technology Adoption Research. Journal of the Association for Information Systems, 8(4), 267-286.

World Economic Forum (WEF) (2009), Global Information Technology Report 2008-2009. Mobility in a Networked World. Ginebra: World Economic Forum

Yi, Y., Wu, Z. y Tung, L. L. (2006), How individual differences influence technology usage behavior? Toward an integrated Framework. The Journal of Computer Information Systems, 46(2), 52-63.

Yousafzai, S. Y., Foxall, G. R. y Pallister, J. G. (2007 a), Technology 
Polis, Revista de la Universidad Bolivariana, Volumen 10, $N^{\circ}$ 30, 2011

acceptance: a meta-analysis of the TAM: Part 1. Journal of Modelling in Management, 2(3), 251-280.

Yousafzai, S. Y., Foxall, G. R. y Pallister, J. G. (2007 b), Technology acceptance: a meta-analysis of the TAM: Part 2. Journal of Modelling in Management, 2(3), 281-304.

Recibido: 12.10 .2010

Aceptado: 20.07.2011 